\title{
Therapeutic Potential of Vortioxetine for Anxious Depression: A Post Hoc Analysis of Data from a Clinical Trial Conducted in Japan
}

\author{
Takeshi Inoue (D) \\ Shinji Fujimoto $\mathbb{D}^{2}$ \\ Tatsuro Marumoto ${ }^{2}$ \\ Tadayuki Kitagawa $^{3}$ \\ Kazuyuki Ishida ${ }^{3}$ \\ Tadashi Nakajima ${ }^{2}$ \\ Yoshiya Moriguchi $\mathbb{D}^{4}$ \\ Keita Fujikawa ${ }^{2}$ \\ Koichiro Watanabe ${ }^{5}$ \\ 'Department of Psychiatry, Tokyo \\ Medical University, Tokyo, Japan; ${ }^{2} J a p a n$ \\ Medical Office, Takeda Pharmaceutical \\ Co., Ltd, Tokyo, Japan; ${ }^{3}$ Takeda \\ Development Center - Japan, Takeda \\ Pharmaceutical Co., Ltd, Osaka, Japan; \\ ${ }^{4}$ Medical Affairs, Lundbeck Japan K.K., \\ Tokyo, Japan; ${ }^{5}$ Department of \\ Neuropsychiatry, Kyorin University \\ School of Medicine, Tokyo, Japan
}

Correspondence: Takeshi Inoue Department of Psychiatry, Tokyo Medical University, 6-7-I Nishishinjuku, Shinjukuku, Tokyo, 160-0023, Japan

Tel +8I-3-3342-6III (ext. 5754)

Fax +8I-3-3340-4499

Email tinoue@tokyo-med.ac.jp
Aim: Antidepressants, including selective serotonin reuptake inhibitors, often elicit a poor response in patients with major depressive disorder (MDD) with significant anxiety symptoms. This study investigated the effects of the multimodal antidepressant vortioxetine in patients with MDD and associated anxiety.

Methods: This was a post hoc analysis of data from an 8-week, randomized, double-blind, placebo-controlled, Phase 3 study of vortioxetine $(10 \mathrm{mg}$ or $20 \mathrm{mg}$ ) in Japanese patients aged 20-75 years with recurrent MDD and a Montgomery-Åsberg Depression Rating Scale (MADRS) score of at least 26. Changes from baseline to week 8 in MADRS total score and Hamilton Depression Rating Scale (HAM-D) anxiety/somatization factor score were assessed in patients with anxious depression (HAM-D anxiety/somatization factor score $\geq 7$ ) and without anxious depression.

Results: Data were available for 489 patients. In patients with anxious depression, the leastsquares (LS) mean difference (95\% confidence interval [CI]) versus placebo in change in MADRS total score was $-3.44(-6.10,-0.77)$ for vortioxetine $10 \mathrm{mg}$ and $-4.51(-7.15$, -1.87 ) for vortioxetine $20 \mathrm{mg}$. In patients with non-anxious depression, the LS mean difference $(95 \% \mathrm{CI})$ versus placebo was $-1.81(-4.71,1.09)$ and $-1.05(-4.00,1.90)$ for vortioxetine $10 \mathrm{mg}$ and $20 \mathrm{mg}$, respectively. Changes from baseline in HAM-D anxiety/ somatization factor score were greater in patients treated with vortioxetine $10 \mathrm{mg}$ or $20 \mathrm{mg}$ than in those treated with placebo.

Conclusion: Vortioxetine may be effective for patients with anxiety symptoms in MDD. Further research is warranted to investigate these effects in a real-world clinical setting.

Clinical Trials Registration: ClinicalTrials.gov identifier for primary study: NCT02389816

Keywords: antidepressant, anxiety, major depressive disorder, serotonin reuptake inhibitor and modulator (S-RIM), vortioxetine

\section{Introduction}

Major depressive disorder (MDD) is a common condition associated with a wide range of psychosomatic symptoms relating to a highly heterogeneous pathophysiology. Although antidepressants and psychotherapy have long been available to treat MDD, antidepressants often elicit a poor response in patients with MDD with significant anxiety symptoms. ${ }^{1}$ Treatment approaches need to take into account each patient's individual symptoms; ${ }^{2}$ however, more evidence is needed to guide optimal drug selection for patients with different MDD symptoms. 
Anxiety symptoms are frequently associated with depressive mood in patients with MDD, regardless of their ethnicity or culture. ${ }^{3}$ Indeed, patients with MDD and anxious distress, a valid specifier for MDD in the Diagnostic and Statistical Manual of Mental Disorders, fifth edition (DSM-V), require intensive treatment because of treatment resistance. ${ }^{4}$ Furthermore, other types of associated anxiety disorders are often comorbid with MDD. ${ }^{5}$ Of all types of MDD with high levels of anxiety symptoms, anxious depression, as determined by the Hamilton Depression Rating Scale (HAM-D) anxiety/somatization factor score, has been the most extensively investigated. ${ }^{6,7}$

Some selective serotonin reuptake inhibitors (SSRIs), approved for the treatment of a subset of anxiety disorders, are considered by Japanese psychiatrists to be the best antidepressants for patients with MDD with anxiety symptoms. ${ }^{2}$ Consistently, SSRIs are shown to have greater efficacy in patients with MDD and high-level anxiety than bupropion. ${ }^{8}$ Nevertheless, the results of the large-scale STAR*D trial demonstrated that anxious depression is associated with a poorer response to treatment with antidepressants, including SSRIs, and a higher burden of associated side effects, than non-anxious depression. ${ }^{9}$ Thus, poor treatment outcomes in anxious depression remain a major issue to be addressed in MDD. ${ }^{10}$

Vortioxetine is a multimodal antidepressant, which not only inhibits 5-HT transporters (SERTs) but also modulates several 5-HT receptors. Vortioxetine was developed as a serotonin reuptake inhibitor and modulator based on the hypothesis that combining $5-\mathrm{HT}_{3} / 5-\mathrm{HT}_{1 \mathrm{~A}}$ receptor antagonism with SERT inhibition could have therapeutic potential for both depressed mood and anxiety. ${ }^{11}$ Through these combined mechanisms, it has been postulated that vortioxetine enhances both glutamate and monoaminergic neurotransmission. ${ }^{12,13}$ Indeed, favorable outcomes have been observed in vortioxetine-treated patients with MDD and high anxiety scale scores. ${ }^{14}$

Vortioxetine is currently prescribed for the treatment of MDD in many countries. In Japan, vortioxetine was approved for use based on the results of a randomized, double-blind, placebo-controlled, phase 3 trial that investigated the safety and efficacy of the drug in Japanese patients with MDD. ${ }^{15}$ The robustness of data collected from the phase 3 trial was the basis for several post hoc analyses, including that discussed here. In other analyses, the authors investigated the therapeutic potential of vortioxetine for anhedonia in depression and early improvement with vortioxetine as a predictor of response and remission in Japanese patients, the results of which have been published in this journal.

The therapeutic potential of vortioxetine on MontgomeryÅsberg Depression Rating Scale (MADRS) total score and other outcomes were evaluated in a post hoc analysis of data from Japanese patients with anxious depression in the phase 3 trial, ${ }^{15}$ the results of which are described herein.

\section{Patients and Methods Study Design}

This was a post hoc analysis of data from a multicenter, randomized, double-blind, placebo-controlled, parallelgroup, phase 3 trial investigating the safety and efficacy of vortioxetine in Japanese patients with MDD (ClinicalTrials.gov identifier: NCT02389816). ${ }^{15}$ The trial was conducted from April 10, 2015 to March 16, 2018. Eligible patients completed a 1-3-week screening period and a single-blind 1-week placebo run-in period to minimize the placebo response. Patients were then randomly assigned (1:1:1) to receive vortioxetine $10 \mathrm{mg}$, vortioxetine $20 \mathrm{mg}$ or placebo once daily for 8 weeks during a double-blind treatment period. Two vortioxetine doses were investigated because both $10 \mathrm{mg}$ and $20 \mathrm{mg}$ demonstrated nominally significant improvements in MADRS total score and response rate, and acceptable tolerability, in the secondary analysis of a randomized, placebocontrolled, multinational, 8-week, Phase 2/3 trial of vortioxetine that included Japanese participants with MDD. ${ }^{16}$

After the treatment period, study drugs were withdrawn, at which point patients entered a 4-week safety follow-up period for the analysis of outcomes of adverse events. All patients provided written informed consent before study entry. Investigations were conducted in accordance with the Declaration of Helsinki and the International Council on Harmonisation tripartite guideline on the ethical principles of Good Clinical Practice. ${ }^{17}$ The clinical study protocol, the investigator's brochure, a sample informed consent form, and other study related documents were reviewed and approved by the local or central Institutional Review Boards of all study sites (Supplemental Information S1).

\section{Patients}

Data for patients in the full analysis set of the primary study were included in this post hoc analysis; these were patients who received at least one dose of the study drug (vortioxetine $10 \mathrm{mg}$, vortioxetine $20 \mathrm{mg}$, or placebo) 
during the 8-week, double-blind, randomized treatment period. Full inclusion, exclusion, and study entry criteria have been described previously. ${ }^{15}$ Briefly, patients were adults aged 20-75 years old and had a primary diagnosis of recurrent MDD according to the DSM-IV text revision (TR) criteria for $296.3 x$, corresponding to F33 in ICD$10,{ }^{18}$ with the current major depressive episode having lasted 3-12 months (inclusive) at the time of enrollment for clear diagnostic certainty and exclusion of possible concurrent mental disorders. Patients were required to have a MADRS total score $\geq 26$ from screening to study drug initiation, to include patients with moderate or severe MDD. Patients were excluded if they had previously experienced a failure to respond to two or more antidepressants prescribed for at least 6 weeks, to include suitable subjects for the evaluation of drug effect, or if they presented with a positive urine drug-screening test result. Patients who were diagnosed with any psychiatric disorder as defined by the DSM-IV-TR and patients who had any substance-induced mood disorder (excluding nicotine- and caffeine-related disorders) were also excluded. Patients with symptoms of anxiety were eligible to participate in the trial; however, patients with MDD and a formal diagnosis of an anxiety disorder (as defined by the DSM-IVTR) were excluded.

In this post hoc study, patients were stratified by baseline HAM-D anxiety/somatization factor score, whereby anxious depression was defined as a HAM-D anxiety/somatization factor score of at least 7 (ie, high levels of anxiety symptoms) and non-anxious depression was defined as a HAM-D anxiety/somatization factor score of less than $7 .^{6,7}$ The anxiety/somatization factor comprised the following subscales from the original 17-item HAM-D (HAM-D17): Q10, Anxiety (psychic); Q11, Anxiety (somatic); Q12, Somatic Symptoms (G.I.); Q13, Somatic Symptoms (general); Q15, Hypochondriasis; and Q17, Insight. ${ }^{7}$

\section{Study Outcomes and Assessments}

The primary outcome of this analysis was change in MADRS total score from baseline to week 8 for patients with anxious depression. Other outcomes included MADRS response and remission rates at week 8 , and the mean change from baseline to week 8 in HAM-D anxiety/ somatization factor score. MADRS response was defined as a decrease of at least $50 \%$ in MADRS total score from baseline, and remission was defined as a decrease in MADRS total score to 10 or lower. Analyses were also performed for other relevant outcomes, including the
MADRS subscale scores, HAM-D17, Digit Symbol Substitution Test (DSST), Sheehan Disability Scale (SDS), and the Perceived Deficit Questionnaire-5 (PDQ-5).

\section{Statistical Analyses}

Patient demographics and baseline characteristics were summarized descriptively ( $\mathrm{n}, \%$, or mean and standard deviation). Vortioxetine was evaluated against placebo for each efficacy outcome in patients with anxious depression and non-anxious depression using an analysis of covariance (ANCOVA) model, with treatment as a fixed effect and the baseline score as a covariate. The point estimates of the differences in the least-squares (LS) means between each treatment group and the placebo group (each treatment group - placebo group) and the two-sided $95 \%$ confidence intervals (CIs) were provided. The last observation carried forward (LOCF) method was used for missing data.

\section{Results}

\section{Patient Demographics and Baseline Characteristics}

Data were available for 489 patients from the original study (full analysis set): 161 of these patients received placebo, 165 received vortioxetine $10 \mathrm{mg}$, and 163 received vortioxetine $20 \mathrm{mg}$. In the primary study, patient demographics and baseline characteristics, including disease severity (MADRS score), were well balanced between treatment groups and have been described previously. ${ }^{15}$

In the post hoc analysis, there were $282(57.7 \%)$ patients with anxious depression and 207 (42.3\%) patients with nonanxious depression. Anxious depression was moderate to severe (MADRS $\geq 26$ and HAM-D $\geq 18$ ). The mean baseline MADRS total scores ranged between 31.4 and 31.5 for patients with anxious depression, and 29.3 and 30.0 for patients with non-anxious depression (Table 1). The distribution of baseline HAM-D anxiety/somatization factor baseline scores showed a broad bell-shaped histogram peaking at 6 (Figure 1), indicating heterogeneous levels of anxiety symptoms among those with moderate or severe MDD.

\section{Efficacy Outcomes}

In patients with anxious depression, the LS mean difference $(95 \% \mathrm{CI})$ versus placebo in change in MADRS total score was $-3.44(-6.10,-0.77)$ and $-4.51(-7.15,-1.87)$ for vortioxetine $10 \mathrm{mg}$ and $20 \mathrm{mg}$, respectively (Figure 2). 
Table I Patient Baseline Characteristics

\begin{tabular}{|l|l|l|l|}
\hline & $\begin{array}{l}\text { Placebo } \\
(\mathbf{n}=161)\end{array}$ & $\begin{array}{l}\text { Vortioxetine } \\
\text { 10 } \mathbf{~ m g ~ ( n = 1 6 5 ) ~}\end{array}$ & $\begin{array}{l}\text { Vortioxetine } \\
\text { 20 } \mathbf{~ m g ~ ( n = 1 6 3 ) ~}\end{array}$ \\
\hline $\begin{array}{l}\text { Mean (SD) MADRS } \\
\text { total score }\end{array}$ & $30.5(3.9)$ & $30.8(3.7)$ & $30.6(3.6)$ \\
$\begin{array}{l}\text { Anxious depression, } \\
\mathrm{n}(\%)\end{array}$ & $91(56.5)$ & $94(57.0)$ & $97(59.5)$ \\
$\begin{array}{l}\text { Mean (SD) MADRS } \\
\text { score }\end{array}$ & $31.5(4.2)$ & $31.4(4.1)$ & $31.4(3.9)$ \\
$\begin{array}{l}\text { Non-anxious } \\
\text { depression, } \mathrm{n}(\%) \\
\text { Mean (SD) MADRS } \\
\text { score }\end{array}$ & $70(43.5)$ & $71(43.0)$ & $66(40.5)$ \\
\hline
\end{tabular}

Notes: Anxious depression was defined by a HAM-D anxiety/somatization factor score $\geq 7$, whereas non-anxious depression was defined by a score $<7$. The HAM-D anxiety/somatization factor score is derived from the sum of the following subscales: Q10, Anxiety (psychic); QII, Anxiety (somatic); QI2, Somatic Symptoms (G.I.); Q I3, Somatic Symptoms (general); Q 15, Hypochondriasis; and Q I7, Insight. Abbreviations: HAM-D, Hamilton Depression Rating Scale; MADRS, Montgomery-Åsberg Depression Rating Scale; SD, standard deviation.

In those with non-anxious depression, the LS mean difference $(95 \% \mathrm{CI})$ versus placebo was $-1.81(-4.71,1.09)$ and $-1.05(-4.00,1.90)$ for vortioxetine $10 \mathrm{mg}$ and $20 \mathrm{mg}$, respectively. Similar trends were seen for the subscales of MADRS (Supplemental Information S2).

In patients with anxious depression, the estimated MADRS response rate $(95 \% \mathrm{CI})$ was $46.8 \%(36.4 \%$, $57.4 \%)$ and $50.5 \%(40.2 \%, 60.8 \%)$ for vortioxetine $10 \mathrm{mg}$ and $20 \mathrm{mg}$, respectively, versus $27.5 \%(18.6 \%, 37.8 \%)$ for placebo (Figure 3). In these same patients, the estimated
MADRS remission rate $(95 \% \mathrm{CI})$ was $28.7 \%$ (19.9\%, $39.0 \%)$ and $33.0 \%(23.8 \%, 43.3 \%)$ for vortioxetine $10 \mathrm{mg}$ and $20 \mathrm{mg}$, respectively, compared with $17.6 \%(10.4 \%$, $27.0 \%$ ) for placebo. Similar MADRS response rates were reported for patients with non-anxious depression receiving vortioxetine $10 \mathrm{mg}$ and $20 \mathrm{mg}$; however, the response rate for those receiving placebo did not differ from the rates reported with vortioxetine treatment. In the same subgroup, the MADRS remission rate was similar for placebo and vortioxetine $20 \mathrm{mg}$, whereas a greater remission rate was reported for vortioxetine $10 \mathrm{mg}$ (Figure 3).

Overall, the LS mean difference $(95 \% \mathrm{CI})$ from placebo in change in HAM-D anxiety/somatization factor score was $-0.69(-1.20,-0.17)$ for vortioxetine $10 \mathrm{mg}$ and $-0.53(-1.05,-0.01)$ for vortioxetine $20 \mathrm{mg}$, which were both statistically significant $(P=0.009$ and $P=0.047$, respectively). In those with anxious depression, the LS mean difference $(95 \% \mathrm{CI})$ versus placebo in change in HAM-D anxiety/somatization score was $-0.82(-1.59$, $-0.04)$ and $-0.97(-1.75,-0.20)$ for vortioxetine $10 \mathrm{mg}$ and $20 \mathrm{mg}$, respectively (Figure 4). In those with nonanxious depression, the LS mean difference $(95 \% \mathrm{CI})$ versus placebo was $-0.49(-1.11,0.12)$ and $0.10(-0.53$, 0.73 ) for vortioxetine $10 \mathrm{mg}$ and $20 \mathrm{mg}$, respectively.

In patients with anxious depression, vortioxetine treatment was associated with an improvement versus placebo in other relevant outcomes, including the HAM-D17, DSST, SDS, and PDQ-5 scores (Supplemental

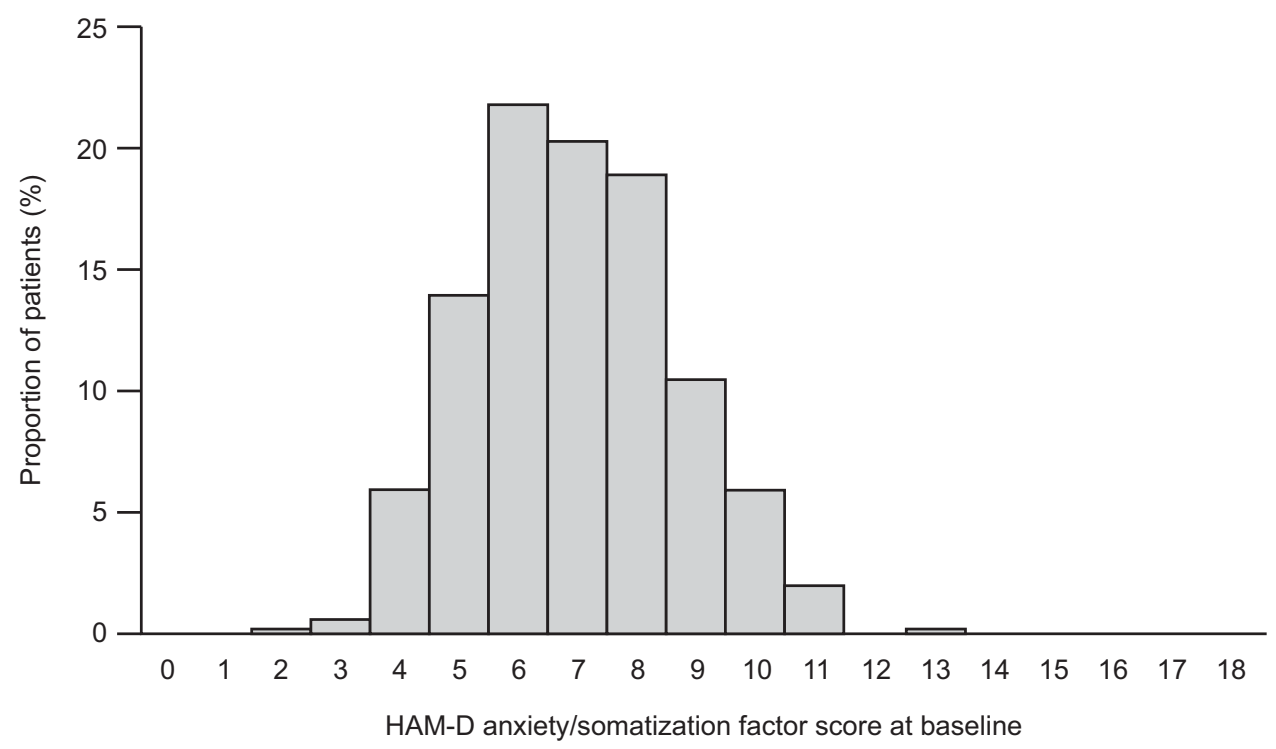

Figure I Distribution of HAM-D anxiety/somatization factor scores at baseline. The HAM-D anxiety/somatization factor score is derived from the sum of the following subscales: Q10, Anxiety (psychic); QII, Anxiety (somatic); Q12, Somatic Symptoms (G.I.); QI3, Somatic Symptoms (general); QI5, Hypochondriasis; and QI7, Insight. Anxious depression was defined by a HAM-D anxiety/somatization factor score $\geq 7$, whereas non-anxious depression was defined by a score $<7$. Abbreviation: HAM-D, Hamilton Depression Rating Scale. 


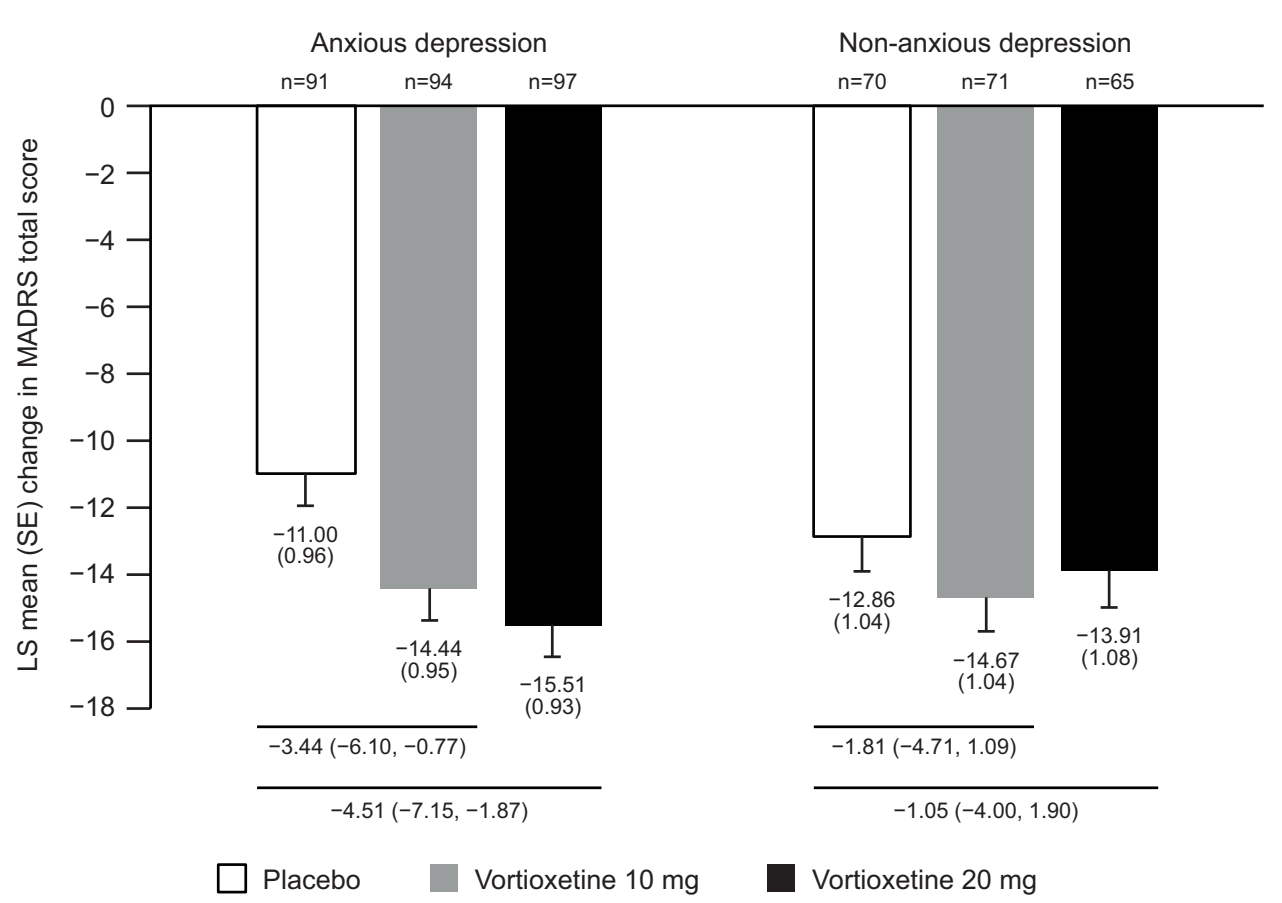

Figure 2 Change in MADRS total score. The bars show the LS mean (SE) change in MADRS total score from baseline to week 8 (LOCF) stratified by anxious depression and non-anxious depression subgroups. The horizontal lines show the LS mean difference $(95 \% \mathrm{Cl})$ between vortioxetine treatment and placebo. Anxious depression was defined by a HAM-D anxiety/somatization factor score $\geq 7$, whereas non-anxious depression was defined by a score $<7$. The HAM-D anxiety/somatization factor score is derived from the sum of the following subscales: QI0, Anxiety (psychic); QI I, Anxiety (somatic); QI2, Somatic Symptoms (G.I.); QI3, Somatic Symptoms (general); QI5, Hypochondriasis; and Q17, Insight.

Abbreviations: Cl, confidence interval; HAM-D, Hamilton Depression Rating Scale; LOCF, last observation carried forward; LS, least-squares; MADRS, MontgomeryÅsberg Depression Rating Scale; SE, standard error.

Information S3). Compared with placebo, improvements in other relevant outcomes were also observed for those with non-anxious depression receiving vortioxetine, except for the HAM-D17 for vortioxetine $20 \mathrm{mg}$ and the DSST for vortioxetine $10 \mathrm{mg}$ and $20 \mathrm{mg}$.

\section{Discussion}

This post hoc study assessed the therapeutic potential of vortioxetine for Japanese patients with MDD with anxiety symptoms. Indeed, MDD is frequently comorbid with a wide range of anxiety disorders, including social anxiety disorder (SAD), generalized anxiety disorder (GAD), obsessive compulsive disorder, and panic disorder, with no particular anxiety disorder predominant in Japan. ${ }^{5}$ Anxious depression has been well documented to reflect multiple aspects of anxiety and may be defined by the HAM-D anxiety/somatization factor score. ${ }^{19}$ In this post hoc analysis, the distribution of HAM-D anxiety/somatization factor scores at baseline indicated heterogeneity in the severity of anxiety symptoms among the patients with MDD who were enrolled in a randomized, double-blind, placebo-controlled, phase 3 trial. ${ }^{15}$ Logistic regression models used in the STAR*D study showed that total
HAM-D anxiety/somatization factor scores can be a good predictor of outcomes. ${ }^{9}$ The HAM-D anxiety/somatization factor score with a threshold of 7 was therefore used in the present study to define patients with or without anxious depression.

Previous studies have shown that vortioxetine can modulate neural transmission and cognitive function via cortical and subcortical regions: in particular, the thalamus. $^{20,21}$ The pulvinar is a higher-order thalamic nucleus that plays an important role in the multisensory processing and emotional response and is extensively connected to the cortex, the superior colliculus, and the amygdala. ${ }^{22-24}$ Thus, pulvinar is well poised to serve as a key neural basis for the antidepressant effect of vortioxetine. Although the role of this neural circuit in anxious depression is unclear and remains to be elucidated, data presented here demonstrate that vortioxetine has therapeutic potential for anxious depression, which has been reported to be poorly responsive to other treatments. ${ }^{1}$ Improvements with vortioxetine therapy were seen in both MADRS total score and the HAM-D anxiety/somatization factor score by which anxious depression was defined. This post hoc study was not designed to compare 

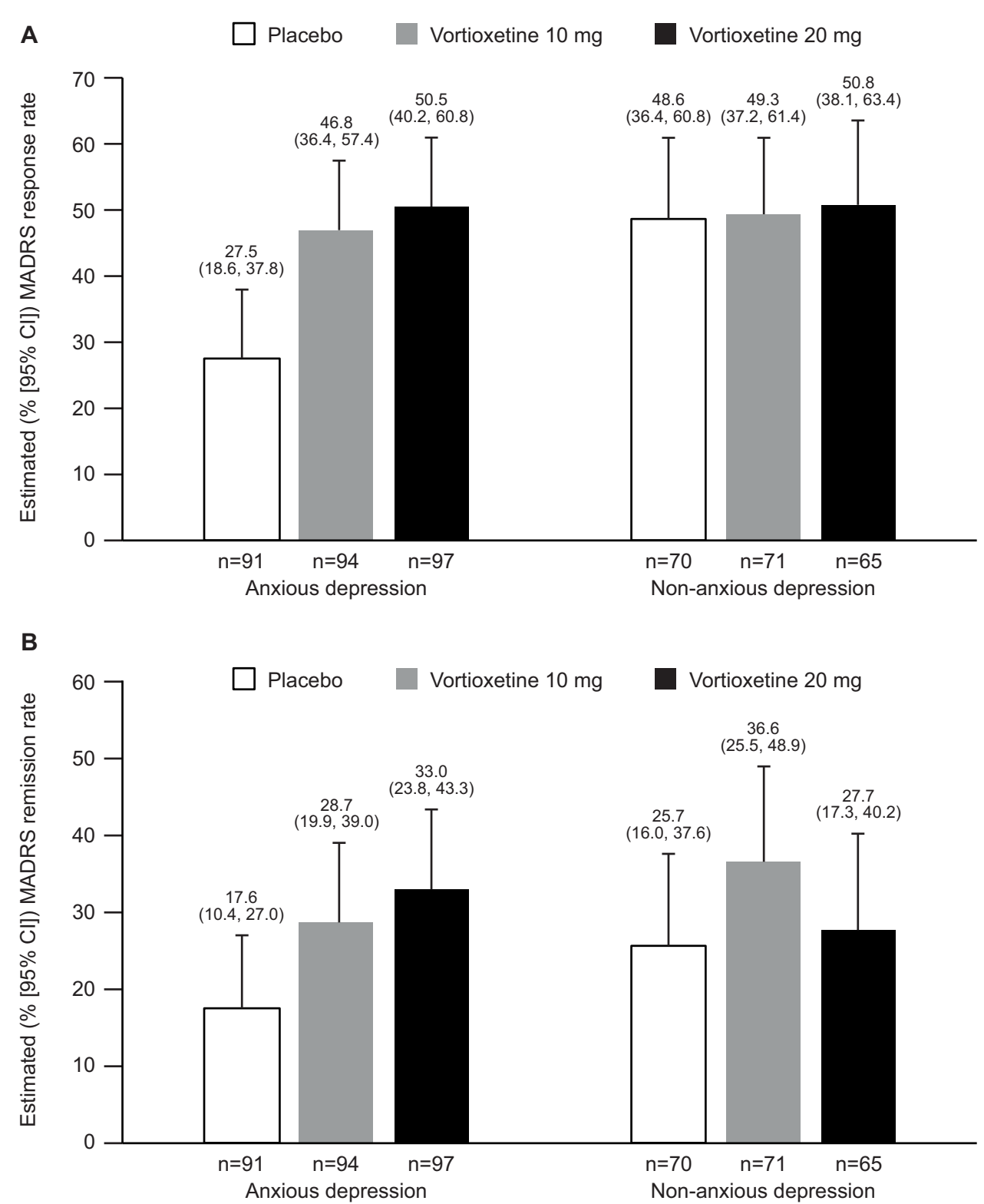

Figure 3 MADRS $(\mathrm{A})$ response rate $(95 \% \mathrm{Cl})$ and $(\mathrm{B})$ remission rate $(95 \% \mathrm{Cl})$ at week 8 (LOCF) stratified by anxious depression and non-anxious depression subgroups. Anxious depression was defined by a HAM-D anxiety/somatization factor score $\geq 7$, whereas non-anxious depression was defined by a score <7. The HAM-D anxiety/ somatization factor score is derived from the sum of the following subscales: Q I0, Anxiety (psychic); QI I, Anxiety (somatic); QI2, Somatic Symptoms (G.I.); QI3, Somatic Symptoms (general); Q15, Hypochondriasis; and QI7, Insight.

Abbreviations: Cl, confidence interval; HAM-D, Hamilton Depression Rating Scale; LOCF, last observation carried forward; MADRS, Montgomery-Åsberg Depression Rating Scale.

efficacy directly in patients with or without anxiety symptoms because there was an insufficient number of patients and the data were obtained from one study and not a pooled analysis. However, vortioxetine was shown to have a numerically greater effect in patients with anxious depression than in those with non-anxious depression across all efficacy outcomes.

Despite the relatively limited efficacy of vortioxetine in GAD shown in a previous study, ${ }^{25}$ the results of this post hoc study suggest that vortioxetine could be a viable option for treating patients with MDD with anxious depression. In a meta-analysis, vortioxetine was found to decrease depression and anxiety scores to a greater extent in patients with MDD with high Hamilton Anxiety Rating Scale (HAM-A) scores than in those with low HAM-A scores. ${ }^{14}$ Vortioxetine has also been shown to have therapeutic potential for patients with MDD comorbid with GAD and SAD. ${ }^{26,27}$ 


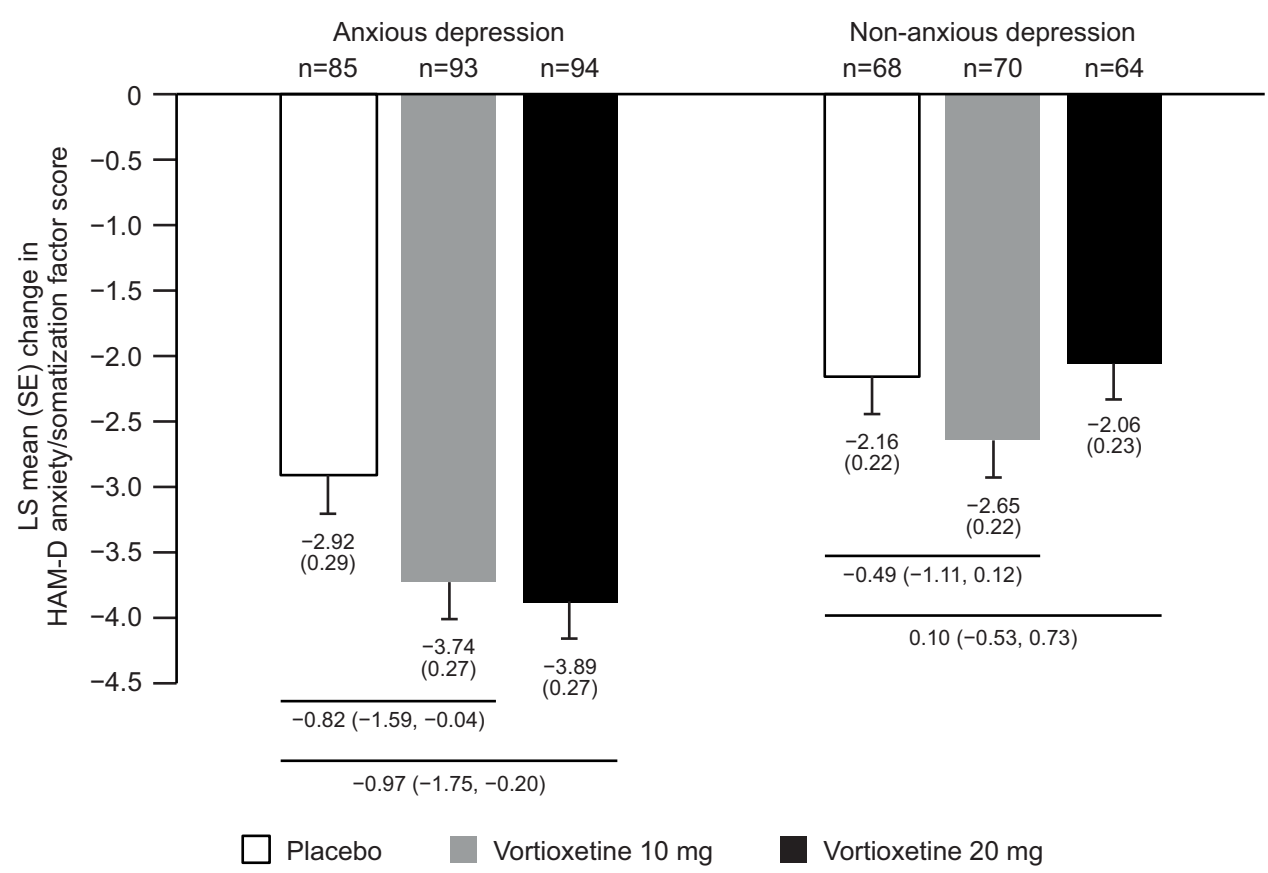

Figure 4 Change in HAM-D anxiety/somatization factor score. The bars show the LS mean (SE) change in HAM-D anxiety/somatization factor score from baseline to week 8 (LOCF) stratified by anxious depression and non-anxious depression subgroups. The horizontal lines show the LS mean difference ( $95 \% \mathrm{Cl}$ ) between vortioxetine treatment and placebo. Anxious depression was defined by a HAM-D anxiety/somatization factor score $\geq 7$, whereas non-anxious depression was defined by a score $<7$. The HAM-D anxiety/somatization factor score is derived from the sum of the following subscales: Q10, Anxiety (psychic); QII, Anxiety (somatic); QI2, Somatic Symptoms (G. I.); QI3, Somatic Symptoms (general); QI5, Hypochondriasis; and QI7, Insight.

Abbreviations: Cl, confidence interval; HAM-D, Hamilton Depression Rating Scale; LOCF, last observation carried forward; LS, least-squares; SE, standard error.

MDD with comorbid anxious symptoms has been consistently associated with poor treatment outcomes. ${ }^{1,28,29}$ SSRIs, rather than traditional anxiolytics, are considered the optimal choice for treating patients with MDD with anxiety symptoms. ${ }^{2,30}$ However, one study found that the number of patients with anxious depression who need to be treated with SSRIs for one patient to benefit (ie, the number needed to treat) was higher than the number of patients with nonanxious depression, particularly among those with severe MDD. ${ }^{10}$ In contrast, a pooled subgroup analysis showed that the serotonin-norepinephrine reuptake inhibitors duloxetine and venlafaxine have similar or higher antidepressant efficacy in patients with anxious depression than in patients with non-anxious depression. ${ }^{31,32}$ Given these findings, antidepressants that only modulate the serotonergic system may have limited efficacy for treating anxious depression.

The results of the present study suggest that vortioxetine may offer a unique clinical profile distinct from SSRIs, perhaps owing to the novel multimodal mechanism of action, including direct 5-HT $1 \mathrm{~A}$ agonism, which could provide an anxiolytic effect similar to that of azapirones such as tandospirone. ${ }^{33}$ Of note, modulation of norepinephrine and dopamine as well as 5-HT may contribute to the anxiolytic effects of antidepressants, and pharmacological interventions targeting these systems could be effective in patients with anxiety and depression. ${ }^{34}$ Vortioxetine increased norepinephrine levels in the ventricular hippocampus and medial prefrontal cortex of rats in a microdialysis study. ${ }^{35}$ This supports our hypothesis that that the diverse pharmacological mechanisms of action of vortioxetine, which, aside from SERT inhibition, SSRIs do not have, may contribute to its effectiveness in anxious depression. Further mechanisms of the anxiolytic effect of vortioxetine remain to be elucidated.

The Clinical Practice Guidelines by the Japanese Society of Mood Disorders stipulate that, in patients with severe MDD, any suitable antidepressant may be initiated at a low dose and titrated upward as soon as possible to its adequate final dose, while monitoring for adverse events. Likewise, the Practice Guidelines by the American Psychiatric Association also recommend the use of the largest tolerable dose of any antidepressant in patients with severe MDD. Contrary to the conventional assumption that antidepressants should be uptitrated when they are found ineffective, a recent systematic review by an international research group reported that antidepressants 
may be most effective when used at a low dose within their approved dose range, suggesting that treatment policy may need to be reconsidered in favor of an alternative antidepressant over dose uptitration. ${ }^{36}$ Terao et al hypothesized that negative feedback by excess SSRI doses through $5-\mathrm{HT}_{1 \mathrm{~A}}$ auto-receptors may account for the bellshaped dose-response curve of SSRIs, which shows decreased efficacy with increasing dose after a certain point. ${ }^{37}$ In this regard, our study results suggest that a higher dose of vortioxetine also may not always be associated with increased efficacy in all patients with depression. Nevertheless, the clinical implications of this study indicate that patients with anxious depression may be treated with vortioxetine, with dose titration up to $20 \mathrm{mg}$.

There were some limitations in the present study. First, the results presented here were derived from a post hoc analysis of data from a clinical trial that did not aim to evaluate the efficacy of vortioxetine in anxious depression or to compare its efficacy in anxious depression and nonanxious depression. Second, all patients included in this post hoc analysis had recurrent MDD, and it remains unclear how recurrent MDD episodes may have affected the psychopathology and manifestation of anxiety symptoms. Therefore, the results of this study may not be readily generalized to all patients with MDD or vortioxetine-treated patients. Third, we were unable to identify any prevalent anxiety symptoms among patients based on the aggregate HAM-D anxiety/somatization factor scores available; therefore, we could not demonstrate the efficacy potential of vortioxetine for any specific anxiety disorder (such as obsessive-compulsive disorder or panic disorder), only for anxious depression in general. The effect of vortioxetine on specific anxiety disorders should be investigated in future research.

\section{Conclusions}

In conclusion, this post hoc analysis indicated that vortioxetine has therapeutic potential for anxious depression as well as for non-anxious depression. Further research is required to investigate the benefit of vortioxetine for patients with MDD with anxiety symptoms in a realworld clinical setting.

\section{Abbreviations}

CI, confidence interval; DSM, Diagnostic and Statistical Manual of Mental Disorders; DSST, Digit Symbol Substitution Test; GAD, generalized anxiety disorder; HAM-
D, Hamilton Depression Rating Scale; LOCF, last observation carried forward; LS, least-squares; MADRS, MontgomeryÅsberg Depression Rating Scale; MDD, major depressive disorder; PDQ-5, Perceived Deficit Questionnaire-5; SD, standard deviation; SE, standard error; SAD, social anxiety disorder; SDS, Sheehan Disability Scale; SERT, 5-HT transporter; SSRI, selective serotonin reuptake inhibitor.

\section{Data Sharing Statement}

The data sets, including the redacted study protocol, redacted statistical analysis plan, and individual participant data supporting the results reported in this article, will be made available by the corresponding author within 3 months from initial request to researchers who provide a methodologically sound proposal. The data will be provided after its de-identification, in compliance with applicable privacy laws, data protection, and requirements for consent and anonymization.

\section{Acknowledgments}

We would like to pay our respects to Dr Yoshinori Watanabe (Himorogi Psychiatric Institute, Tokyo, Japan), who passed away in August of 2021 shortly before submission of this manuscript. We are grateful for his contributions as an investigator in the primary study and the development of this manuscript. Y.W. received speaker's honoraria from Janssen Pharmaceutical K.K., Meiji Seika Pharma Co., Ltd, Mitsubishi Tanabe Pharma Corp., Mochida Pharmaceutical Co., Ltd, Otsuka Pharmaceutical Co., Ltd, Pfizer Japan Inc., Sumitomo Dainippon Pharma Co., and Takeda Pharmaceutical Co., Ltd.

We are grateful to current and former employees of Takeda Pharmaceutical Co., Ltd. and Lundbeck Japan for their contributions to the study: Akira Nishimura and Kiyofumi Sasai (executed the primary clinical trial); Takafumi Akimoto and Aya Makita (supported the design of the analysis); and Isao Inada, Akihiro Takano, and Manami Imazaki (supported the interpretation of the results). Medical writing support was provided by Cara Kingston, $\mathrm{PhD}$, and Svetha Sankar, BSc, BVMS, of Oxford PharmaGenesis, Melbourne Australia, funded by Takeda Pharmaceutical Co., Ltd.

\section{Author Contributions}

All authors made a significant contribution to the work reported, whether that is in study conception, study design, execution, acquisition of data, analysis and interpretation, 
or in all these areas; took part in drafting, revising or critically reviewing the article; gave final approval of the version to be published; agreed on the journal to which the article has been submitted; and agree to be accountable for all aspects of the work.

\section{Funding}

This study was funded by Takeda Pharmaceutical Co., Ltd.

\section{Disclosure}

T.I. served as medical officer of the primary study; has received personal fees from Daiichi Sankyo, Janssen Pharmaceuticals, Lilly, Mochida Pharmaceutical Co., Ltd, MSD, Taisho Toyama Pharmaceutical, Takeda Pharmaceutical Co., Ltd, and Yoshitomiyakuhin; has received grants from Astellas, Eisai, Shionogi Inc., and Tsumura; has received grants and personal fees from Kyowa Pharmaceutical Industry, Meiji Seika Pharma Co., Ltd, Mitsubishi Tanabe Pharma Corp., Novartis Pharma, Otsuka Pharmaceutical Co., Ltd, Pfizer, and Sumitomo Dainippon Pharma; and is a member of the advisory boards of Mitsubishi Tanabe Pharma Corp., Novartis Pharma, and Pfizer. S.F., T.M., T.K., K.I., T.N., and K.F. are employees of Takeda Pharmaceutical Co., Ltd. Y.M. is an employee of Lundbeck Japan K.K. K. W. has received manuscript fees or speaker's honoraria from Astellas, Daiichi Sankyo, GlaxoSmithKline, Janssen Pharmaceuticals, Kyowa Pharmaceutical Industry, Lilly, Meiji Seika Pharma Co., Ltd, Mitsubishi Tanabe Pharma Corp., MSD, Otsuka Pharmaceutical Co., Pfizer, Shionogi Inc., Sumitomo Dainippon Pharma Co., Ltd, Takeda Pharmaceutical Co., Ltd, and Yoshitomi; has received research and grant support from Astellas, Daiichi Sankyo, Eisai, Meiji Seika Pharma Co., Ltd, Mitsubishi Tanabe Pharma Corp., MSD, Otsuka Pharmaceutical Co., Ltd, Pfizer, and Shionogi Inc.; is a consultant for Eisai, Janssen Pharmaceutical, Kyowa Pharmaceutical, Lilly, Mitsubishi Tanabe Pharma Corp., Otsuka Pharmaceutical Co., Ltd, Pfizer, Sumitomo Dainippon Pharma Co., Ltd, and Taisho Toyama Pharmaceutical; and is a consultant and advisory board member for Takeda Pharmaceutical Co. Ltd, Lundbeck Japan, and Viatris, outside the submitted work. The authors report no other conflicts of interest in this work.

\section{References}

1. Gaspersz R, Nawijn L, Lamers F, et al. Patients with anxious depression: overview of prevalence, pathophysiology and impact on course and treatment outcome. Curr Opin Psychiatry. 2018;31:17-25. doi:10.1097/YCO.0000000000000376
2. Sakurai H, Uchida H, Kato M, et al. Pharmacological management of depression: Japanese expert consensus. J Affect Disord. 2020;26 6:626-632. doi:10.1016/j.jad.2020.01.149

3. Kessler RC, Sampson NA, Berglund P, et al. Anxious and non-anxious major depressive disorder in the World Health Organization World Mental Health Surveys. Epidemiol Psychiatr Sci. 2015;24(3):210-226. doi:10.1017/S2045796015000189

4. American Psychiatric Association. Diagnostic and Statistical Manual of Mental Disorders. 5th ed. Arlington (VA): American Psychiatric Publishing; 2013.

5. Inoue T, Kimura T, Inagaki Y, et al. Prevalence of comorbid anxiety disorders and their associated factors in patients with bipolar disorder or major depressive disorder. Neuropsychiatr Dis Treat. 2020;16: 1695-1704. doi:10.2147/NDT.S246294

6. Fava M, Rosenbaum JF, Hoog SL, et al. Fluoxetine versus sertraline and paroxetine in major depression: tolerability and efficacy in anxious depression. $J$ Affect Disord. 2000;59(2):119-126. doi:10.1016/S0165-0327(99)00131-7

7. Fava M, Alpert JE, Carmin CN, et al. Clinical correlates and symptom patterns of anxious depression among patients with major depressive disorder in STAR*D. Psychol Med. 2004;34:1299-1308. doi:10.1017/S0033291704002612

8. Papakostas G, Stahl S, Krishen A, et al. Efficacy of bupropion and the selective serotonin reuptake inhibitors in the treatment of major depressive disorder with high levels of anxiety (anxious depression): a pooled analysis of 10 studies. $J$ Clin Psychiatry. 2008; 69:1287-1292. doi:10.4088/JCP.v69n0812

9. Fava M, Rush AJ, Alpert JE, et al. Difference in treatment outcome in outpatients with anxious versus nonanxious depression: a STAR *D report. Am J Psychiatry. 2008;165:342-351. doi:10.1176/appi.ajp.20 07.06111868

10. Papakostas GI, Fan H, Tedeschini E. Severe and anxious depression: combining definitions of clinical sub-types to identify patients differentially responsive to selective serotonin reuptake inhibitors. Eur Neuropsychopharmacol. 2012;22:347-355. doi:10.1016/j.euroneuro. 2011.09.009

11. Bang-Andersen B, Ruhland T, Jørgensen M, et al. Discovery of 1-[2-(2,4-dimethylphenylsulfanyl)phenyl]piperazine (Lu AA21004): a novel multimodal compound for the treatment of major depressive disorder. J Med Chem. 2011;54(9):3206-3221. doi:10.1021/jm101459g

12. Riga MS, Sánchez C, Celada P, et al. Involvement of 5-HT3 receptors in the action of vortioxetine in rat brain: focus on glutamatergic and GABAergic neurotransmission. Neuropharmacology. 2016;108: 73-81. doi:10.1016/j.neuropharm.2016.04.023

13. Stahl SM. Modes and nodes explain the mechanism of action of vortioxetine, a multimodal agent (MMA): modifying serotonin's downstream effects on glutamate and GABA (gamma amino butyric acid) release. CNS Spectr. 2015;20(4):331-336. doi:10.1017/ S1092852915000334

14. Baldwin DS, Florea I, Jacobsen PL, et al. A meta-analysis of the efficacy of vortioxetine in patients with major depressive disorder (MDD) and high levels of anxiety symptoms. J Affect Disord. 2016;206:140-150. doi:10.1016/j.jad.2016.07.015

15. Inoue T, Sasai K, Kitagawa T, et al. Randomized, double-blind, placebo-controlled study to assess the efficacy and safety of vortioxetine in Japanese patients with major depressive disorder. Psychiatry Clin Neurosci. 2020;74:140-148. doi:10.1111/pen.12956

16. Nishimura A, Aritomi Y, Sasai K, et al. Randomized, double-blind, placebo-controlled 8-week trial of the efficacy, safety, and tolerability of 5,10 , and $20 \mathrm{mg} /$ day vortioxetine in adults with major depressive disorder. Psychiatry Clin Neurosci. 2018;72:64-72. doi:10.1111/pcn.12565

17. International Conference on Harmonisation of Technical Requirements for Registration of Pharmaceuticals for Human Use. ICH harmonized tripartite guideline: guideline for Good Clinical Practice. J Postgrad Med. 2001;47(1):45-50. 
18. World Health Organization. Recurrent depressive disorder International Statistical Classification of Diseases and Related Health Problems 10th Revision (ICD-10); 2019. Available from: https://icd. who.int/browse10/2019/en\#F33. Accessed November 32021.

19. Ionescu DF, Niciu MJ, Henter ID, et al. Defining anxious depression: a review of the literature. CNS Spectr. 2013;18:252-260. doi:10. 1017/S1092852913000114

20. Laursen B, Bundgaard CH, Graversen C, et al. Acute dosing of vortioxetine strengthens event-related brain activity associated with engagement of attention and cognitive functioning in rats. Brain Res. 2017;1664:37-47. doi:10.1016/j.brainres.2017.03.024

21. Okada M, Matsumoto R, Yamamoto Y, et al. Effects of subchronic administrations of vortioxetine, lurasidone, and escitalopram on thalamocortical glutamatergic transmission associated with serotonin 5-ht7 receptor. Int J Mol Sci. 2021;22:1351. doi:10.3390/ijms 22031351

22. Chou XL, Fang Q, Yan L, et al. Contextual and cross-modality modulation of auditory cortical processing through pulvinar mediated suppression. Elife. 2020;9:e54157. doi:10.7554/eLife.54157

23. Fang Q, Chou XL, Peng B, et al. A differential circuit via retino-colliculo-pulvinar pathway enhances feature selectivity in visual cortex through surround suppression. Neuron. 2020;105 (2):355-69.e6. doi:10.1016/j.neuron.2019.10.027

24. Ibrahim LA, Mesik L, Ji XY, et al. Cross-modality sharpening of visual cortical processing through layer-1-mediated inhibition and disinhibition. Neuron. 2016;89:1031-1045. doi:10.1016/j.neuron.20 16.01 .027

25. Slee A, Nazareth I, Bondaronek P, et al. Pharmacological treatments for generalised anxiety disorder: a systematic review and network meta-analysis. Lancet. 2019;393(10173):768-777. doi:10.1016/ S0140-6736(18)31793-8

26. Alvarez E, Perez V, Dragheim M, et al. A double-blind, randomized, placebo-controlled, active reference study of Lu AA21004 in patients with major depressive disorder. Int $J$ Neuropsychopharmacol. 2012;15(5):589-600. doi:10.1017/S1461145711001027

27. Liebowitz MR, Careri J, Blatt K, et al. Vortioxetine versus placebo in major depressive disorder comorbid with social anxiety disorder. Depress Anxiety. 2017;34(12):1164-1172. doi:10.1002/da.22702

28. Wu Z, Chen J, Yuan C, et al. Difference in remission in a Chinese population with anxious versus nonanxious treatment-resistant depression: a report of OPERATION study. J Affect Disord. 2013;150(3):834-839. doi:10.1016/j.jad.2013.03.012
29. Wiethoff K, Bauer M, Baghai TC, et al. Prevalence and treatment outcome in anxious versus nonanxious depression: results from the German Algorithm Project. J Clin Psychiatry. 2010;71 (8):1047-1054. doi:10.4088/JCP.09m05650blu

30. Bandelow B, Sher L, Bunevicius R, et al. Guidelines for the pharmacological treatment of anxiety disorders, obsessive-compulsive disorder and posttraumatic stress disorder in primary care. Int J Psychiatry Clin Pract. 2012;16(2):77-84. doi:10.3109/13651 501.2012.667114

31. Nelson JC. Anxiety does not predict response to duloxetine in major depression: results of a pooled analysis of individual patient data from 11 placebo-controlled trials. Depress Anxiety. 2010;27 (1):12-18. doi:10.1002/da.20632

32. Lyndon GJ, Prieto R, Wajsbrot DB, et al. Efficacy of venlafaxine extended release in major depressive disorder patients: effect of baseline anxiety symptom severity. Int Clin Psychopharmacol. 2019;34:110-118. doi:10.1097/YIC.0000000000000256

33. Lin J, Su Y, Wang C, et al. Effects of tandospirone augmentation in major depressive disorder patients with high anxiety: a multicenter, randomized, parallel-controlled, open-label study. J Psychiatr Res. 2018;99:104-110. doi:10.1016/j.jpsychires.20 18.01.020

34. Goddard AW, Ball SG, Martinez J, et al. Current perspectives of the roles of the central norepinephrine system in anxiety and depression. Depress Anxiety. 2010;27:339-350. doi:10.1002/da.20642

35. Pehrson AL, Cremers T, Bétry C, et al. Lu AA21004, a novel multimodal antidepressant, produces regionally selective increases of multiple neurotransmitters - a rat microdialysis and electrophysiology study. Eur Neuropsychopharmacol. 2013;23:133-145. doi:10.1016/j. euroneuro.2012.04.006

36. Furukawa TA, Cipriani A, Cowen PJ, et al. Optimal dose of selective serotonin reuptake inhibitors, venlafaxine, and mirtazapine in major depression: a systematic review and dose-response meta-analysis. Lancet Psychiatry. 2019;6:601-609. doi:10.1016/S2215-0366(19) 30217-2

37. Terao T, Ishii N, Hirakawa H, et al. Is the bell-shaped dose-response curve of the selective serotonin reuptake inhibitor due to 5-HT1A auto-receptors? Med Hypotheses. 2020;140:109681. doi:10.1016/j. mehy.2020.109681
Neuropsychiatric Disease and Treatment

\section{Publish your work in this journal}

Neuropsychiatric Disease and Treatment is an international, peerreviewed journal of clinical therapeutics and pharmacology focusing on concise rapid reporting of clinical or pre-clinical studies on a range of neuropsychiatric and neurological disorders. This journal is indexed on PubMed Central, the 'PsycINFO' database and CAS, and

\section{Dovepress}

is the official journal of The International Neuropsychiatric Association (INA). The manuscript management system is completely online and includes a very quick and fair peer-review system which is all easy to use. Visit http://www.dovepress.com/testimonials.php to read real quotes from published authors. 